\title{
The Comparison of Long-Term Outcome Between Patients with Single and Multiple Coronary Chronic Total Occlusions After Percutaneous Coronary Intervention
}

\author{
Miaomiao Cao, Bolin Li, Qian Li (D), Chaofeng Sun \\ Department of Cardiovascular Medicine, The First Affiliated Hospital of Xi'an Jiaotong University, Xi'an, Shaanxi, 7I006I, People's Republic of China \\ Correspondence: Chaofeng Sun, Department of Cardiovascular Medicine, The First Affiliated Hospital of Xi'an Jiaotong University, No. 277 Yanta \\ West Road, Xi'an, 7I006I, People's Republic of China, Email cfsunI23@I26.com
}

\begin{abstract}
Background: Rapid advancements in percutaneous coronary intervention (PCI) have improved the outcome of chronic coronary total occlusions (CTOs); however, data regarding the difference in long-term outcomes in stable coronary artery disease (CAD) patients with single and multiple CTOs who underwent PCI are scarce. This study aimed to compare the long-term outcomes of patients with multiple CTOs and single CTO after PCI.

Methods: This study cohort included stable CAD patients who were diagnosed with CTO and treated with PCI from a single center. The primary endpoint was all-cause death.

Results: We retrospectively reviewed 670 consecutive patients with CTO-PCI. Among them, 539 patients had a single CTO, and 131 (19.7\%) patients had multiple (at least two) CTOs. CTO revascularization was achieved in 470 (70.1\%) patients. After a median follow-up duration of 33.7 months, the cumulative all-cause mortality $(p=0.037)$ and cardiac mortality $(p=0.003)$ were higher in patients with multiple CTOs than in those with a single CTO. In the multivariable model, multiple CTOs and left ventricular ejection fraction (LVEF) less than 40\% were independent predictors for cardiac death (adjusted hazard ratio (HR) $2.53 ; \mathrm{P}=0.013$ and adjusted HR 3.95; $\mathrm{P}<0.001$ ), while age older than 65 and LVEF less than $40 \%$ were independent predictors for all-cause death in CTO-PCI patients (adjusted hazard ratio (HR) 1.84; $\mathrm{P}=0.035$ and adjusted HR 2.54; $\mathrm{P}=0.001$ ).

Conclusion: In CTO-PCI patients, long-term survival was associated with multiple CTOs, age and LVEF, but not with CTO revascularization.
\end{abstract}

Keywords: coronary chronic total occlusion, percutaneous coronary intervention, clinical outcomes, cardiac death

\section{Introduction}

Chronic coronary total occlusions (CTOs) are characterized by total occlusion in the coronary artery with a TIMI flow grade of 0 for at least 3 months. Approximately $18-52 \%$ of patients with known or suspected coronary artery disease who underwent coronary angiography are reported to have CTO. ${ }^{1}$ Up to $12 \%$ of patients who underwent coronary angiography and $23 \%$ of CTO patients had at least two CTOs. ${ }^{2}$

Previous studies reported that CTO is associated with worsened cardiac function and prognosis. Percutaneous coronary intervention (PCI) is a well-accepted technique for CTO revascularization, but has a higher rate of procedure failure, and restenosis than in nonoccluded arteries. ${ }^{3}$ Multiple CTOs make the procedure more challenging for operators, and few studies have investigated the clinical outcome of multiple CTOs versus single CTO treated with PCI. In addition, a few studies have indicated that CTO-PCI improves symptoms, and may prolong the survival of CTO patients, but whether the CTO number influences the clinical outcomes of CTO patients who undergo PCI is poorly understood. 
Hence, we compared the baseline characteristics and clinical outcomes between patients with single and multiple CTOs, aiming to investigate the impact of CTO numbers on the survival of patients after CTO-PCI.

\section{Methods}

\section{Study Population and Definition}

Information on this study population has been reported previously. ${ }^{4}$ We retrospectively reviewed 858 consecutive CTO patients who underwent PCI at the First Affiliated Hospital of Xi'an Jiaotong University from June 2013 to October 2017. Patients with diagnosis of acute coronary syndrome (ACS) were excluded. CTO-PCI was performed by highly experienced operators following the current guidelines. ${ }^{5}$ The CTO was defined as total occlusion in the coronary artery with a TIMI flow grade of 0 for at least 3 months. Multiple CTOs was defined as CTO lesions located in $\geq 2$ vessels. Multivessel disease was defined as stenosis with a diameter greater more than $70 \%$ in $\geq 1$ coronary arteries in addition to the occluded artery. Revascularization was defined as stenosis with a residual diameter of less than $30 \%$ in all occluded vessels with a TIMI flow grade of 3 . The degree of diameter stenosis was determined by visual assessment on angiography from highly experienced operators. All baseline characteristics, surgical information and laboratory examinations were collected from the electronic medical record system.

\section{Follow-Up and Study Outcomes}

Telephone contacts were prospectively made between 2018 and 2019. The primary endpoint was all-cause mortality. Cardiac death was defined as death within 7 days after myocardial infarction (MI) or stroke, death associated with cardiovascular interventions within 7 days after PCI, or unexpected death presumed to be due to ischemic cardiovascular disease and occurring within $24 \mathrm{~h}$ after the onset of symptoms without evidence of other cause. Major adverse cardiac events (MACEs) were defined as the composite of cardiac death, nonfatal acute MI, revascularization and stroke during follow-up.

\section{Statistical Analysis}

All data analyses and visualizations were performed using SPSS software, version 18.0 (SPSS, Inc.) and GraphPad Prism 6 (GraphPad Software, La Jolla, CA, U.S.A.). Continuous variables are presented as the mean \pm SD or median (interquartile range), while categorical variables are presented as numbers and percentages. The distribution patterns of continuous variables were evaluated by the Kolmogorov-Smirnov test. Independent $t$-tests, Mann-Whitney $U$-tests and chi-squared tests were applied to compare two groups of continuous and categorical variables. Cumulative survival curves were generated by the Kaplan-Meier method and compared by the Log rank test. Cox proportional hazards regression models were performed by the forward stepwise method to identify the independent predictors for all-cause death and cardiac death. We used bivariate correlation coefficients to identify collinearity. When the two variables are significantly correlated $(\mathrm{P}<0.05)$, only one was selected in the final model. The selection was based on the results of the univariate analysis and clinical relevance. Variables with a P-value less than 0.1 in the univariate analysis and several variables that were considered clinically relevant were entered into the multivariable model. Given the number of events available, the following variables were included: age $>65$, sex, prior MI, left anterior descending artery (LAD), left circumflex artery (LCX), right coronary artery (RCA), multiple CTOs, CTO revascularization, renal dysfunction, and left ventricular ejection fraction (LVEF) $<40 \%$. A two-sided P value less than 0.05 was regarded as statistically significant.

\section{Results}

\section{Patients' Characteristics}

A total of 858 patients with CTO lesions were screened from June 2013 to October 2017. Of these patients, 97 patients diagnosed with ACS at admission and 91 patients lost to follow-up were excluded. The final study cohort included 670 patients with 818 CTO lesions who underwent PCI. CTO revascularization was achieved in 470 (70.1\%) patients. According to the number of CTO vessels, patients were divided into two groups: the single CTO ( $\mathrm{n}=539)$ group and the multiple CTO (n=131) group (Figure 1). 


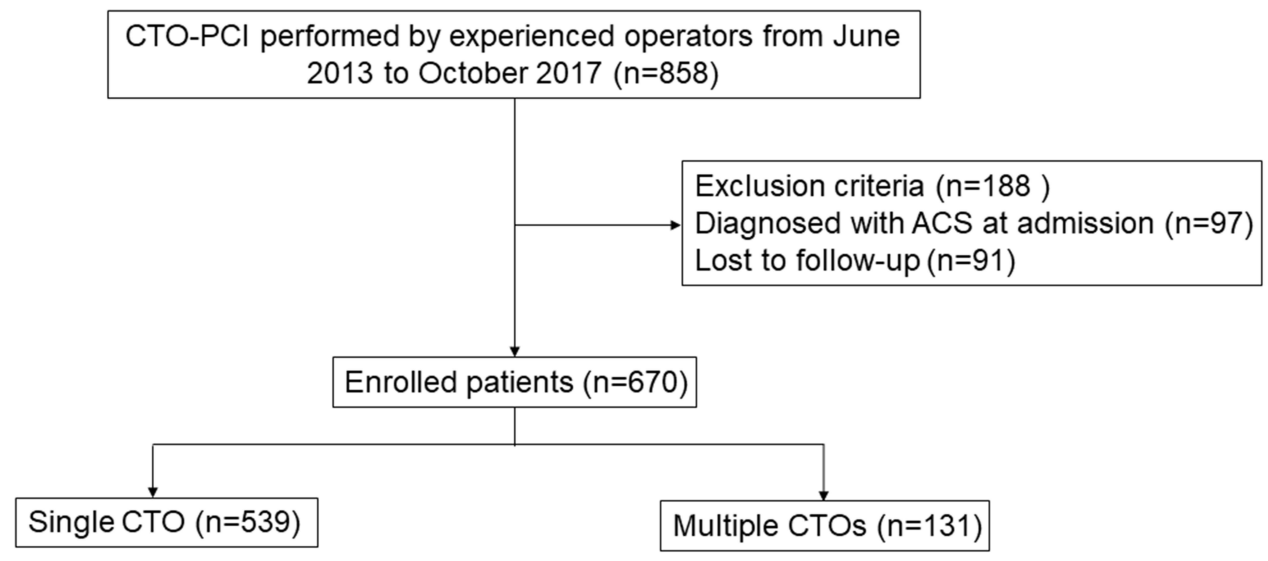

Figure I Study flowchart.

Abbreviations: CTO, chronic total occlusion; $\mathrm{PCl}$, percutaneous intervention; $\mathrm{ACS}$, acute coronary syndrome.

Table 1 shows the clinical characteristics of the two groups. Approximately $83.6 \%$ of CTO-PCI patients were male, with a mean age of 65 years. CTO lesions were most commonly observed in the right coronary artery (47.5\%), followed by LAD (40.3\%) and LCX (30.9\%). The prevalence of multivessel disease in patients with CTO-PCI was $85.7 \%$. The medications for patients with CTO-PCI during the follow-up period mainly included aspirin (85.4\%), clopidogrel (45.0\%), statins (71.7\%), ACEIs/ARBs (27.5\%), beta-blockers $(62.3 \%)$, etc. Compared with the single CTO group, patients with multiple CTOs were older, and had a lower estimated glomerular filtration rate and LVEF (Table 1).

\section{Clinical Outcomes}

After a median follow-up period of 33.7 months (interquartile range 22.5 to 48.8 months), sixty patients died, of whom thirty-one died because of cardiovascular causes. Recurrent MI and stroke were observed in 27 (4.0\%) and 29 (4.3\%) patients, respectively. Approximately 476 (71.0\%) patients had a rehospitalization (Table 2). Kaplan-Meier survival curves estimated the cumulative all-cause mortality, cardiac mortality, and MACE for the two groups in Figure 2, showing that patients with multiple CTOs had higher cumulative all-cause mortality $(\mathrm{p}=0.037)$ and cardiac mortality $(\mathrm{p}=0.003)$. Patients with CTO-PCI did not have a survival benefit from revascularization for cardiac death in either the single or multiple CTOs group. Significant differences in cardiac mortality existed between the single or multiple CTOs group either in revascularization ( $\mathrm{p}=0.018$ ) or no revascularization group $(\mathrm{p}=0.029)$ (Figure 3$)$. In the univariate analysis, multiple CTOs, revascularization, and $\mathrm{LVEF}<40 \%$ were found to be significant risk factors of cardiac mortality (Table S1), while age $>65$, and $\mathrm{LVEF}<40 \%$ were the significant risk factors of all-cause mortality (Table S2). The multivariable analysis revealed that multiple CTOs and LVEF $<40 \%$ were independent predictors for cardiac mortality, while age $>65$ and $\mathrm{LVEF}<40 \%$ were independent predictors for all-cause mortality (Table 3). The results of subgroup analyses are provided in Table S3).

\section{Discussion}

Our study presented new data to explore the impact on the long-term outcome of multiple CTOs versus single CTOs in stable CAD patients after PCI. The main findings are as follows: (1) Mortality was higher in patients with multiple CTOs than in those with a single CTO. (2) Long-term survival in CTO-PCI patients was associated with multiple CTOs, age, and LVEF, but not with CTO revascularization.

In the present study, $19.6 \%$ of CTO patients were identified with multiple CTOs. CTO lesions were most commonly seen in the right coronary artery, and more prevalent in males and older patients. These results are consistent with the previous study. ${ }^{6,7}$ CTO refers to thrombotic occlusion that leads to blocking of the coronary artery, which results in myocardial ischemia, the most severe subtype of CAD. As reported, the prevalence of prior MI in CTO patients was $42 \%$ to $68 \%$, which is higher than that in patients without CTO. Most patients with CTO had multivessel coronary disease. ${ }^{8}$ Evidence has showed that the presence of CTO is associated with symptomatic angina, a high risk of malignant arrhythmias and mortality. ${ }^{9,10}$ Multiple CTOs were responsible for more mortality than a single CTO or non-CTO artery 
Table I Baseline Characteristics for Patients with Single and Multiple CTOs

\begin{tabular}{|c|c|c|c|c|}
\hline & Total $(n=670)$ & Single СTO $(n=539)$ & Multiple CTOs $(n=|3|)$ & $P$ value \\
\hline Age, years & $65.37 \pm 9.99$ & $64.94 \pm 10.18$ & $67.12 \pm 9.02$ & 0.017 \\
\hline Age $>65$ & $369(55.1)$ & $283(52.5)$ & $86(65.6)$ & 0.007 \\
\hline Male & $560(83.6)$ & $453(84.0)$ & $107(81.7)$ & 0.512 \\
\hline BMI, $\mathrm{kg} / \mathrm{m}^{2}$ & $24.55 \pm 3.39$ & $24.61 \pm 3.32$ & $24.28 \pm 3.68$ & 0.365 \\
\hline Prior MI & $217(32.4)$ & I7I (3I.7) & $46(35.1)$ & 0.457 \\
\hline Hypertension & $372(55.5)$ & $304(56.4)$ & $68(51.9)$ & 0.353 \\
\hline Diabetes mellitus & $238(35.5)$ & $189(35.1)$ & $49(37.4)$ & 0.616 \\
\hline Previous stroke & $68(10.1)$ & $53(9.8)$ & $15(11.5)$ & 0.582 \\
\hline Peripheral vascular disease & $2(0.3)$ & $2(0.40)$ & $0(0.00)$ & 0.483 \\
\hline Current smoker & $332(49.6)$ & $27 \mid(50.3)$ & $61(46.6)$ & 0.446 \\
\hline Drunk & $149(22.2)$ & $123(22.6)$ & $26(19.8)$ & 0.463 \\
\hline NYHA status & & & & 0.106 \\
\hline I & $37(6.0)$ & $31(6.2)$ & $6(5.3)$ & \\
\hline II & $450(72.8)$ & $367(72.8)$ & $83(72.8)$ & \\
\hline III & $98(15.9)$ & $84(16.7)$ & $14(12.3)$ & \\
\hline IV & $33(5.3)$ & $22(4.4)$ & $\mathrm{II}(9.6)$ & \\
\hline Stent NO. & $2.0(2.0-3.0)$ & $2.0(2.0-3.0)$ & $2.0(2.0-3.0)$ & 0.048 \\
\hline \multicolumn{5}{|l|}{ СТO located in } \\
\hline LAD & $270(40.3)$ & $191(35.4)$ & $79(60.3)$ & $<0.001$ \\
\hline LCX & 207(30.9) & III (20.6) & $96(73.8)$ & $<0.001$ \\
\hline $\mathrm{RCA}$ & $318(47.5)$ & $223(41.4)$ & $95(72.5)$ & $<0.001$ \\
\hline LM & $3(0.4)$ & $2(0.4)$ & $\mathrm{I}(0.8)$ & 0.546 \\
\hline Other & $\mathrm{II}(\mathrm{I} .6)$ & $7(1.3)$ & $4(3.1)$ & 0.156 \\
\hline СТО revascularization & $470(70.1)$ & $372(69.0)$ & $98(74.8)$ & 0.194 \\
\hline Multivessel disease & $574(85.7)$ & $44 I(82.1)$ & $131(100.0)$ & $<0.001$ \\
\hline h-CRP, mg/l & $1.4(1.1-1.8)$ & I.4 (I.I-I.7) & I.4 (I.2-2.0) & 0.145 \\
\hline eGFR (MDRD), $\mathrm{mL} /\left(\mathrm{min}^{*} \mathrm{l} .73^{2}\right)$ & $99.30 \pm 30.00$ & $100.46 \pm 29.54$ & $94.54 \pm 31.48$ & 0.043 \\
\hline Renal dysfunction & $57(8.5)$ & $4 I(7.6)$ & $16(12.2)$ & 0.093 \\
\hline $\mathrm{TC}, \mathrm{mmol} / \mathrm{l}$ & $3.69(3.07-4.33)$ & $3.66(3.05-4.33)$ & $3.74(3.16-4.33)$ & 0.430 \\
\hline $\mathrm{TG}, \mathrm{mmol} / \mathrm{l}$ & $1.42(1.01-2.06)$ & $1.42(1.01-2.06)$ & $1.41(1.00-2.09)$ & 0.905 \\
\hline LDL-C, mmol/l & $2.09(1.60-2.66)$ & $2.09(1.58-2.66)$ & $2.07(1.66-2.67)$ & 0.721 \\
\hline LVEF, \% & $60(45-67)$ & $60(47-67)$ & $49(40-64)$ & $<0.001$ \\
\hline LVEF $<40 \%$ & $103(16.3)$ & $7 \mid(14.0)$ & $32(25.8)$ & 0.001 \\
\hline \multicolumn{5}{|l|}{ Medications during follow-up } \\
\hline Aspirin & $486(85.4)$ & $402(86.1)$ & $84(82.4)$ & 0.334 \\
\hline Clopidogrel & $256(45.0)$ & $204(43.7)$ & $52(51.0)$ & 0.180 \\
\hline Ticagrelor & $46(8.1)$ & $37(7.9)$ & $9(8.8)$ & 0.762 \\
\hline Statin & $407(71.7)$ & 331 (70.9) & $76(75.2)$ & 0.377 \\
\hline ACEI/ARB & $184(27.5)$ & $154(33.2)$ & $30(29.7)$ & 0.498 \\
\hline Beta-blocker & $354(62.3)$ & $287(6 \mid .5)$ & $67(66.3)$ & 0.359 \\
\hline
\end{tabular}

Note: Values are mean $\pm S D$, number (\%), or median (interquartile range).

Abbreviations: CTO, chronic total occlusion; BMI, body mass index; MI, myocardial infarction; LAD, left anterior descending artery; LCX, left circumflex artery; RCA, right coronary artery; h-CRP, high sensitive C-reactive protein; eGFR, estimated glomerular filtration rate; TC, total cholesterol; TG, triglycerides; LDL-C, low-density lipoprotein; LVEF, left ventricular ejection fraction; ACEI, angiotensin-converting enzyme inhibitor; ARB, angiotensin receptor blocker.

in patients with ST-elevation myocardial infarction. ${ }^{10}$ Our findings also suggest that multiple CTOs conferred a higher mortality than single CTO in patients with CTO-PCI. Moreover, multiple CTOs was the independent predictor of cardiac death. The possible explanations are as follows. On one hand, well-developed collateral vessels can maintain myocardial viability and reduce ischemic burden by providing retrograde flow to the myocardial perfusion area of a CTO. However, in the setting of multiple CTOs, inadequate flow may bring more ischemia burden. On the other hand, the inferior survival of patients with multiple CTOs might partially be attributed to the higher risk clinical characteristics compared 
Table 2 Clinical Outcome of Patients with Single and Multiple CTOs

\begin{tabular}{|l|l|l|l|l|}
\hline Events & Total (n=670) & Single CTO (n=539) & Multiple CTO (n=13I) & P value \\
\hline MACE & I3I(I9.6) & $99(18.6)$ & $32(24.4)$ & 0.131 \\
All-cause death & $60(9.0)$ & $42(7.8)$ & $18(13.7)$ & 0.033 \\
Cardiac death & $3 I(4.6)$ & $18(3.3)$ & $13(9.9)$ & $0.00 I$ \\
Re-admission & $476(7 \mid .0)$ & $384(7 \mid .2)$ & $92(70.2)$ & 0.818 \\
Recurrent MI & $27(4.0)$ & $23(4.3)$ & $4(3.1)$ & 0.526 \\
Stroke & $29(4.3)$ & $27(5.0)$ & $2(1.5)$ & 0.079 \\
\hline
\end{tabular}

Note: Values are number (\%).

Abbreviations: CTO, chronic total occlusion; $\mathrm{PCl}$, percutaneous intervention; $\mathrm{MACE}$, major adverse cardiac events; $\mathrm{MI}$, myocardial infarction.

with the single CTO group, including older age, a higher rate of left ventricular dysfunction and other comorbidities, such as renal dysfunction, which might indirectly influence myocardial viability, and collateral circulation. ${ }^{11,12}$ Further studies are needed to confirm this finding and explore the possible mechanisms.
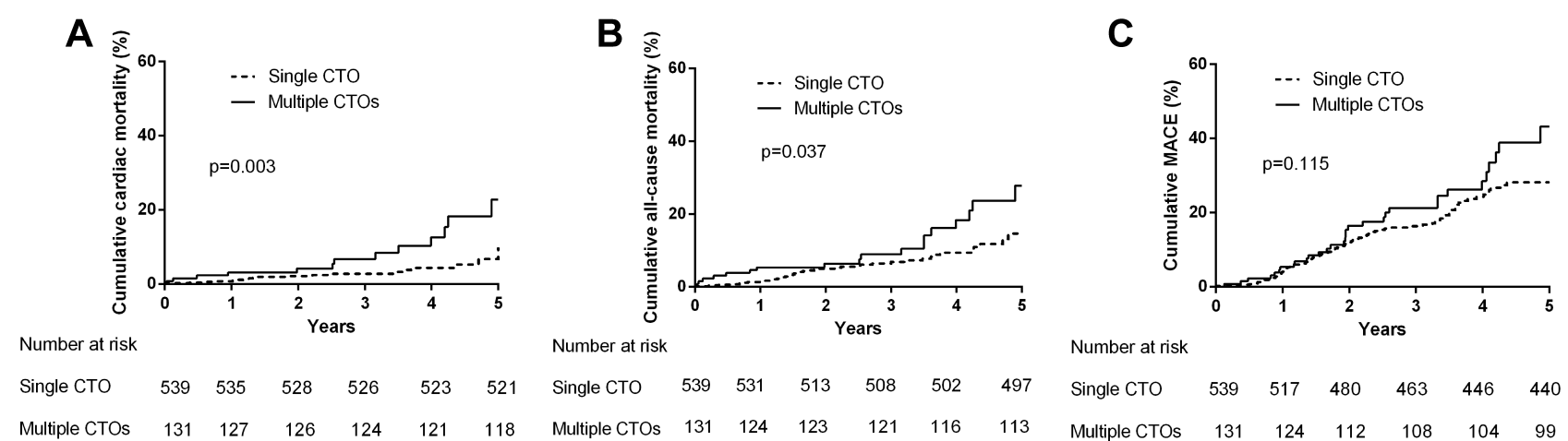

$\begin{array}{lllllllllllllllllllll}\text { Single СтО } & 539 & 535 & 528 & 526 & 523 & 521 & \text { Single CTO } & 539 & 531 & 513 & 508 & 502 & 497 & \text { Single Сто } & 539 & 517 & 480 & 463 & 446 & 440\end{array}$

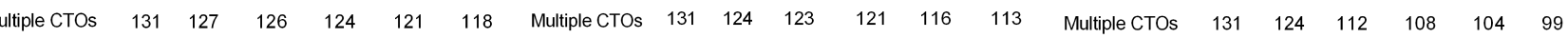

Figure 2 Kaplan-Meier curve of cumulative incidence of (A) cardiac mortality of single and multiple CTOs groups; (B) all-cause mortality of single and multiple CTOs groups; (C) MACE of single and multiple CTOs groups.

Abbreviations: CTO, chronic total occlusion; MACE, major adverse cardiovascular events.

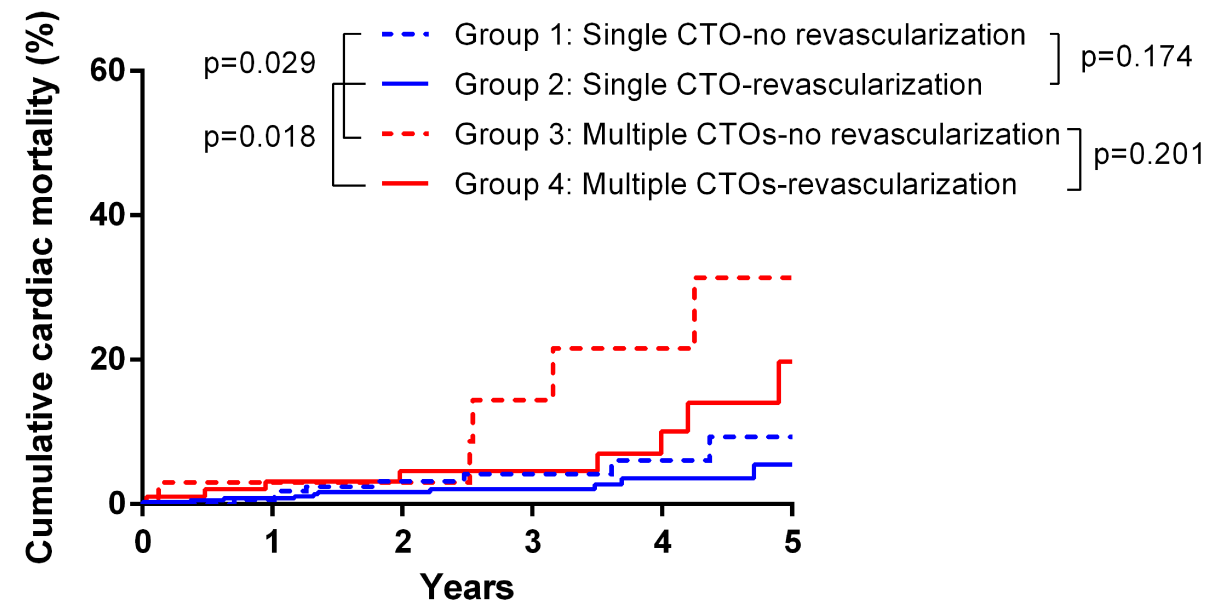

$\begin{array}{lcccccc}\text { Number at risk } & & & & & \\ \text { Group 1 } & 167 & 166 & 162 & 161 & 160 & 159 \\ \text { Group 2 } & 372 & 369 & 366 & 365 & 363 & 362 \\ \text { Group 3 } & 33 & 32 & 32 & 30 & 29 & 28 \\ \text { Group 4 } & 98 & 95 & 94 & 94 & 92 & 90\end{array}$

Figure 3 Kaplan-Meier curve of cumulative cardiac mortality of revascularization or no-revascularization in single and multiple CTOs groups. Abbreviation: CTO, chronic total occlusion. 
Table 3 Independent Predictors of All-Cause Death, Cardiac Death in All Patients with CTO-PCI

\begin{tabular}{|l|l|l|l|}
\hline & HR & $\mathbf{9 5 \%} \mathbf{~ C l}$ & P value \\
\hline $\begin{array}{l}\text { Independent predictors of all-cause death } \\
\text { Age }>65\end{array}$ & 1.842 & $1.044-3.252$ & $\begin{array}{l}0.035 \\
\text { LVEF }<40 \%\end{array}$ \\
$\begin{array}{l}\text { Independent predictors of cardiac death } \\
\text { Multiple CTO }\end{array}$ & 2.540 & $1.480-4.362$ & 0.001 \\
LVEF $<40 \%$ & 2.530 & $1.217-5.261$ & 0.013 \\
& 3.950 & $1.908-8.175$ & $<0.001$ \\
\hline
\end{tabular}

Abbreviations: CTO, chronic total occlusion; PCI, percutaneous intervention; LVEF, left ventricular ejection fraction; $\mathrm{HR}$, hazard ratio; $\mathrm{Cl}$, confidence interval; other abbreviations as in Table I.

The discussion regarding the benefits of CTO-PCI is still under debate. In our results, no difference in cardiac mortality was observed between successful versus unsuccessful CTO-PCI, while other study reported an increased risk of cardiac death and malignant ventricular arrhythmias in patients with unsuccessful CTO-PCI. ${ }^{13}$ A meta-analysis reviewed 25 observational studies including 28,486 CTO patients who underwent the PCI procedure, suggesting that successful CTO-PCI is associated with a lower incidence of mortality and MI, improvement of angina, and fewer demands of subsequent coronary artery bypass surgery. ${ }^{14}$ Another two randomized trials of IMPACTOR-CTO (Impact on Inducible Myocardial Ischemia of Percutaneous Coronary Intervention versus Optimal Medical Therapy in Patients with Right Coronary Artery Chronic Total Occlusion) and EuroCTO (A Randomized Multicentre Trial to Evaluate the Utilization of Revascularization or Optimal Medical Therapy for the Treatment of Chronic Total Coronary Occlusions) confirmed the benefit of symptomatic improvement of successful CTO-PCI, but not for survival. ${ }^{15,16}$ In terms of multiple CTOs, an observational study found that complete CTO revascularization was associated with improved survival. ${ }^{11}$ However, both single CTO group and multiple CTOs group did not show improved survival after revascularization in our results. Interestingly, patients with multiple CTOs still had a higher cumulative cardiac mortality than those with a single CTO either in revascularization group $(\mathrm{p}=0.018)$ or no revascularization group $(\mathrm{p}=0.029)$. The possible explanation was that the patients with multiple CTOs were older and had more comorbidities.

Given the rapid advances in the equipment and techniques, CTO-PCI is recommended with a level of Class IIa in the current guidelines. ${ }^{5}$ However, many issues interfere with the selection of therapeutic strategies in patients with CTO, such as the success rate of the procedure, a risk/benefit analysis or comorbidities. Moreover, powerful evidence is absent when deciding on the revascularization approach in the special CTO population, including multiple CTOs, left ventricular dysfunction or elderly patients. ${ }^{11,17-19}$ Hence, more randomized controlled trials regarding these questions are necessary.

Our study has several limitations. First, it was an observational study from a single center, so selection bias may exist. Our study only included hospitalized patients who were highly suspected of having coronary artery disease, which may overlook CTO patients without symptomatic angina. Moreover, we excluded patients with ACS at admission, thus the results may not be suitable for this group. Second, the conclusions about the efficacy of CTO-PCI cannot be drawn from this non-randomized study. Third, because we retrospectively collected data by using medical record system, a few factors, such as myocardial viability, revascularization technique, the number of attempts, major complications, lesion length, calcification, and collateral circulation could not be retrieved and analysed in this study.

\section{Conclusion}

In stable CAD patients with CTO-PCI, multiple CTOs conferred higher mortality than a single CTO. Long-term survival was independently associated with age, left ventricular ejection fraction and multiple CTOs, but not with CTO revascularization. These findings might help to guide the optimal treatment for CTO patients. 


\section{Abbreviations}

$\mathrm{CAD}$, coronary artery disease; CTO, chronic coronary total occlusion; PCI, percutaneous coronary intervention; ACS, acute coronary syndrome; MI, myocardial infarction; MACEs, major adverse cardiac events; LAD, left anterior descending artery; LCX, left circumflex artery; RCA, right coronary artery; LVEF, left ventricular ejection fraction; HR, hazard ratio; CI, confidence interval.

\section{Data Sharing Statement}

The data described in this study are available upon reasonable request from the corresponding author.

\section{Ethics Approval and Consent to Participate}

This study didn't include identified personal information. Thus, a waiver of individual consent and ethics were reviewed and approved by the First Affiliated Hospital of Xi'an Jiaotong University ethics committee (Ethical approval number: XJTU1AF2012LSK-313). The study was conducted in accordance with the Declaration of Helsinki (as revised in 2013).

\section{Funding}

There is no funding to report.

\section{Disclosure}

The authors have no conflicts of interest to declare.

\section{References}

1. Galassi AR, Brilakis ES, Boukhris M, et al. Appropriateness of percutaneous revascularization of coronary chronic total occlusions: an overview. Eur Heart J. 2016;37(35):2692-2700b. doi:10.1093/eurheartj/ehv391

2. Christofferson RD, Lehmann KG, Martin GV, et al. Effect of chronic total coronary occlusion on treatment strategy. Am J Cardiol. 2005;95 (9):1088-1091. doi:10.1016/j.amjcard.2004.12.065

3. Stone GW, Reifart NJ, Moussa I, et al. Percutaneous recanalization of chronically occluded coronary arteries: a consensus document: part II. Circulation. 2005;112(16):2530-2537. doi:10.1161/CIRCULATIONAHA.105.583716

4. Li B, Rong J, Wang B, et al. Cystatin C-based renal function in predicting the long-term outcomes of chronic total occlusion after percutaneous coronary intervention. Front Cardiovasc Med. 2020;7:586181. doi:10.3389/fcvm.2020.586181

5. Windecker S, Kolh P, Alfonso F, et al.; Authors/Task Force members. 2014 ESC/EACTS Guidelines on myocardial revascularization: the Task Force on Myocardial Revascularization of the European Society of Cardiology (ESC) and the European Association for Cardio-Thoracic Surgery (EACTS) Developed with the special contribution of the European Association of Percutaneous Cardiovascular Interventions (EAPCI). Eur Heart J. 2014;35(37):2541-2619. doi:10.1093/eurheartj/ehu278

6. Flores-Umanzor EJ, Cepas-Guillen PL, Caldentey G, et al. Sex-based differences in chronic total occlusion management and long-term clinical outcomes. Int J Cardiol. 2020;319:46-51. doi:10.1016/j.ijcard.2020.05.090

7. Flores-Umanzor EJ, Vazquez S, Cepas-Guillen P, et al. Impact of revascularization versus medical therapy alone for chronic total occlusion management in older patients. Catheter Cardiovasc Interv. 2019;94(4):527-535. doi:10.1002/ccd.28163

8. Stone GW, Kandzari DE, Mehran R, et al. Percutaneous recanalization of chronically occluded coronary arteries: a consensus document: part I. Circulation. 2005;112(15):2364-2372. doi:10.1161/CIRCULATIONAHA.104.481283

9. Raja V, Wiegn P, Obel O, et al. Impact of chronic total occlusions and coronary revascularization on all-cause mortality and the incidence of ventricular arrhythmias in patients with ischemic cardiomyopathy. Am J Cardiol. 2015;116(9):1358-1362. doi:10.1016/j.amjcard.2015.07.057

10. Bataille Y, Dery JP, Larose E, et al. Prevalence, predictors and clinical impact of unique and multiple chronic total occlusion in non-infarct-related artery in patients presenting with ST-elevation myocardial infarction. Heart. 2012;98(23):1732-1737. doi:10.1136/heartjnl-2012-302376

11. Danzi GB, Valenti R, Migliorini A, et al. Percutaneous coronary intervention for multiple chronic total occlusions. Am J Cardiol. 2013;112 (12):1849-1853. doi:10.1016/j.amjcard.2013.08.012

12. Hoebers LP, Claessen BE, Dangas GD, et al. Contemporary overview and clinical perspectives of chronic total occlusions. Nat Rev Cardiol. 2014;11(8):458-469. doi:10.1038/nrcardio.2014.74

13. Godino C, Giannattasio A, Scotti A, et al. Risk of cardiac and sudden death with and without revascularisation of a coronary chronic total occlusion. Heart. 2019;105(14):1096-1102. doi:10.1136/heartjnl-2018-314076

14. Christakopoulos GE, Christopoulos G, Carlino M, et al. Meta-analysis of clinical outcomes of patients who underwent percutaneous coronary interventions for chronic total occlusions. Am J Cardiol. 2015;115(10):1367-1375. doi:10.1016/j.amjcard.2015.02.038

15. Obedinskiy AA, Kretov EI, Boukhris M, et al. The IMPACTOR-CTO trial. JACC Cardiovasc Interv. 2018;11(13):1309-1311. doi:10.1016/j. jcin.2018.04.017

16. Werner GS, Martin-Yuste V, Hildick-Smith D, et al. A randomized multicentre trial to compare revascularization with optimal medical therapy for the treatment of chronic total coronary occlusions. Eur Heart J. 2018;39(26):2484-2493. doi:10.1093/eurheartj/ehy220

17. Ybarra LF, Rinfret S. Why and how should we treat chronic total occlusion? Evolution of state-of-the-art methods and future directions. Can J Cardiol. 2020. doi:10.1016/j.cjca.2020.10.005 
18. Guo L, Meng S, Lv H, et al. Long-term outcomes of successful recanalization compared with optimal medical therapy for coronary chronic total occlusions in patients with and without left ventricular systolic dysfunction. Front Cardiovasc Med. 2021;8:654730. doi:10.3389/ fcvm.2021.654730

19. Tian T, Guan C, Gao L, et al. Predictors for adverse outcomes of patients with recanalized chronic total occlusion lesion. Eur J Clin Invest. 2021;51 (2):e13368. doi:10.1111/eci.13368

\section{Publish your work in this journal}

The Journal of Inflammation Research is an international, peer-reviewed open-access journal that welcomes laboratory and clinical findings on the molecular basis, cell biology and pharmacology of inflammation including original research, reviews, symposium reports, hypothesis formation and commentaries on: acute/chronic inflammation; mediators of inflammation; cellular processes; molecular mechanisms; pharmacology and novel anti-inflammatory drugs; clinical conditions involving inflammation. The manuscript management system is completely online and includes a very quick and fair peer-review system. Visit http://www.dovepress.com/testimonials.php to read real quotes from published authors.

Submit your manuscript here: https://www.dovepress.com/journal-of-inflammation-research-journal 